\title{
Gastroptosis due to Gastric Outlet Obstruction Secondary to Duodenal Tumor: Glenard's Disease Revisited
}

\author{
Muhammad Begawan Bestaria Melissa Chandra ${ }^{a}$ \\ Ignatius Ronaldi Joewono ${ }^{a}$ Dolvy Girawan ${ }^{a}$ Rizky Andhikab \\ Yudi Wahyudia Siti Aminah Abdurachman ${ }^{a}$ \\ aDivision of Gastroenterohepatology, Department of Internal Medicine, Hasan Sadikin \\ General Hospital-Faculty of Medicine University of Padjadjaran, Bandung, Indonesia; \\ ${ }^{b}$ Division of Nephrology and Hypertension, Department of Internal Medicine, Hasan Sadikin \\ General Hospital-Faculty of Medicine University of Padjadjaran, Bandung, Indonesia
}

\author{
Keywords \\ Gastroptosis · Gastric outlet obstruction · Glenard's disease · Duodenal tumor
}

\section{Abstract}

Currently, gastroptosis is rarely reported, and the actual prevalence is unknown. Similarly, the possible predisposing factor and cause remain unclear. A 69-year-old had melena for 1 week, and other symptoms were left upper abdominal pain, nausea, and vomiting especially postprandially that was persistent for several months with no response to medication. The nasogastric tube produced yellowish discharge and dark-colored undigested material. The CT scan showed obstruction of the gastroduodenal junction and gastroptosis. Endoscopy revealed a gastric lumen that was extended inferiorly and an occluded pyloric ring by a bleeding mass protruding from the duodenum. We report the first case of gastroptosis caused by gastric outlet obstruction secondary to duodenal tumor.

\section{Karger $\stackrel{2}{\circ}$}




\section{Introduction}

Glenard's disease also called visceroptosis describes downward displacement of visceral organs. Gastroptosis, a more specific description, is a condition in which the stomach is displaced inferiorly [1]. In the past, gastroptosis was frequently diagnosed. However, only a few cases have been reported recently, and the actual prevalence of gastroptosis is unknown.

\section{Case Report/Case Presentation}

A 69-year-old female, presented in the emergency room with left upper quadrant abdominal pain, aggravated by meals. She also complained of black tarry stool twice a week prior to admission with frequent defecation 2-3 times a day for the past week. There were also symptoms of nausea, vomiting, and weight loss of $10 \mathrm{~kg}$ in the past 3 months, and she was hospitalized at another hospital, with no improvement of symptoms after 1 month of medication.

On physical examination, the patient appeared malnourished. A mass was palpated in the abdominal left upper quadrant, measuring approximately $10 \mathrm{~cm}$ which disappeared thereafter. Abdominal X-ray showed no obstruction, perforation, or peritonitis. Abdominal ultrasound findings suggested gastric dilatation.

Blood examination showed low hemoglobin $7.7 \mathrm{gr} / \mathrm{dL}$ and hypokalemia $2.5 \mathrm{mmol} / \mathrm{L}$. Other examinations, such as urea, creatinine, sodium, chloride, prothrombin time, activated partial thromboplastin time, and liver function tests were within normal limits. The patient was initially diagnosed with upper gastrointestinal bleeding due to gastric cancer and hypokalemia. She was then administered a high-dose intravenous proton pump inhibitor, $12 \mathrm{~h}$ intravenous potassium, blood transfusion, nasogastric tube placement, intravenous fluid, and parenteral nutrition via right femoral central line. She was also scheduled for upper gastrointestinal endoscopy and colonoscopy.

During the hospital stay, the patient's nasogastric tube produced yellowish discharge with foul-smelling dark-colored semisolid material. This prompted us to perform abdominal CT scan due to suspicion of bowel obstruction, which revealed obstruction at the gastroduodenal junction with gastric enlargement and gastroptosis (Fig. 1). Upon endoscopy, the gastric lumen extended inferiorly and was located at a lower position than normal, which is consistent with the CT scan findings of gastroptosis. The gastric lumen was filled with yellow liquid with semisolid dark-colored content, and gastric mucosa and vascularization were normal. The pyloric ring was occluded by a mass from the duodenum pushing toward the pylorus, so the scope could not pass through the mass. Blood was leaking through the pyloric ring possibly originating from the duodenal mass (Fig. 2a). Biopsy samples were taken although the mass itself was beyond the biopsy forceps which only revealed chronic gastritis. Endoscopic ultrasound was performed using a radial scope, which showed an irregular heterogeneous hypoechoic mass at the duodenum, measuring $1.44 \times 1.21 \mathrm{~cm}$ (Fig. $2 \mathrm{~b}$ ). Unfortunately the radial scope was not suited for fine-needle biopsy. The diagnosis was Glenard's disease presenting as gastroptosis secondary to duodenal tumor with pyloric stenosis and signs of gastric outlet obstruction (GOO).

Surgery consultation was performed, and a plan to operate on this patient was made. However, after discussion and informed consent regarding the need for surgery the family refused and opted for comfort care instead. The patient passed away due to hospital-acquired pneumonia shortly thereafter, during the same admission.

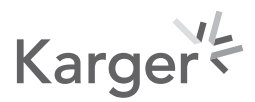


Bestari et al.: Gastroptosis due to Gastric Outlet Obstruction Secondary to Duodenal Tumor

Fig. 1. Coronal view of an abdominal CT scan showing an enlarged stomach (red bracket) with the cardiac portion located in the normal position, greater curvature reaching the iliac crest (blue arrow), and the pyloric portion located below the normal position (yellow arrow).
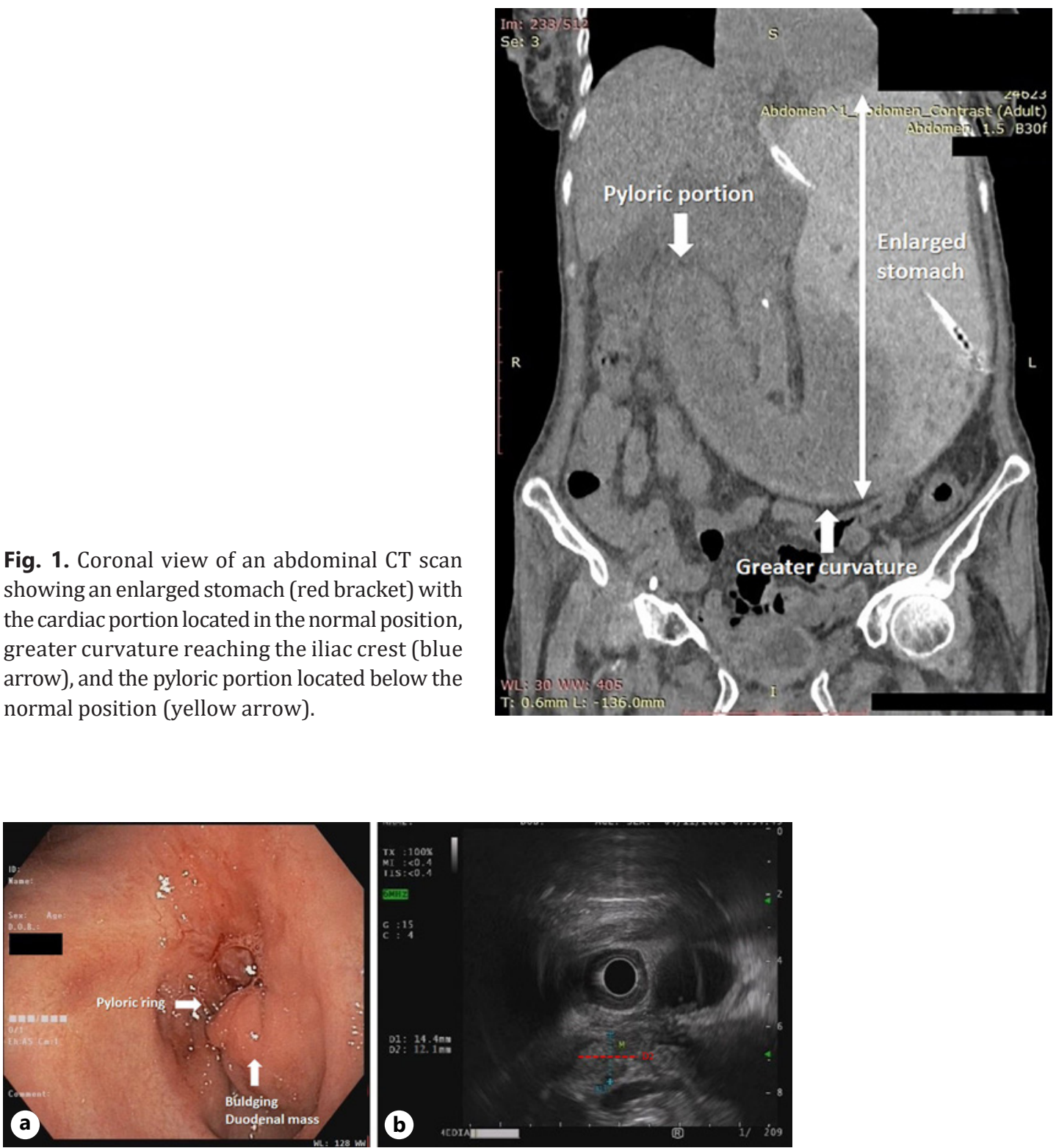

Fig. 2. a Upper GI endoscopy showing a duodenal mass obstructing the pyloric ring (black arrow). The mass pushed the pylorus, and noticeable blood leaked from the duodenum through the pyloric ring (blue arrow). b Endoscopic ultrasound image using radial scope showing a duodenal mass with a heterogeneous hypoechoic pattern with unclear margins measuring $1.44 \times 2.1 \mathrm{~cm}$ (red arrow).

\section{Discussion and Conclusion}

This is the first reported case of a duodenal mass causing gastroptosis secondary to GOO. A similar case was reported in 2019 wherein lumen-occupying protruding duodenal ulcers caused obstruction [2]. However, no gastroptosis was reported. Glenard's disease is the term used for downward displacement of the visceral organ, also known as visceroptosis. "Gastroptosis" is used when the dropping of the viscera involves the stomach, "enteroptosis" involves the intestines, "coloptosis" involves the colon, "hepatoptosis" involves the liver, and "nephroptosis" involves the kidney [3]. 
When the greater curvature is permanently located below the umbilicus, the diagnosis of gastroptosis can be made [1]. Currently, there is no clear indicator for the degree of prolapse that is clinically significant. A study in Japan reported that gastroptosis was observed in $12 \%$ of men and $43 \%$ of females, and most of the patients were underweight [4]. The most common complaints for gastroptosis were bloating, nausea, and vomiting, especially postprandially $[5,6]$. Reported complications from gastroptosis are back pain and gastroparesis [1, 7]. In this case, all the common symptoms were present. However, the patient complained that these symptoms were severe and intractable to mediations, which made us speculated that the severity of the symptoms was caused by gastroparesis resulted from the combination of mechanical obstruction from the GOO and aggravated by gastroptosis. Gastroparesis also increases the risk of aspiration pneumonia. Whether the risk of pneumonia further increases when the cause of gastroptosis was both GOO and gastroptosis remains unknown.

The retained gastric content drained from the nasogastric tube may be caused by the accumulation of undigested food and blood that leaked from the pylorus, which created a foul-smelling and dark-colored material. Hassan, et al. [8] reported similar findings from patient with GOO due to adult idiopathic hypertrophic pyloric stenosis. In GOO, a large amount of food debris is retained because only gastric juice promotes digestion in contrast with smallbowel obstruction, in which food is exposed to pancreatic enzymes that play an important role in digesting food debris [9].

Gastroptosis is caused by relaxation of the ligaments and mesenteries due to the weight of the organ; another possible cause for this condition is diseases originating from the intestine and/or stomach which lead to the weakening and exhaustion of the ligaments [1]. In this case, trapped gastric content increased the weight of the stomach, and it was aggravated by malnourishment and duodenal tumor which weakened the ligament and mesenteries. As the condition progressed, the trapped gastric content dropped further downward and the opening of the pylorus became narrower, creating a viscous cycle. Previous studies reported that in clinical gastroptosis, the cardiac portion is never displaced [3]. Although on abdominal X-ray the obstruction was undetected because barium study was not done, an abdominal CT scan (Fig. 1) revealed that the cardia was located in the normal position, whereas the pylorus was displaced inferiorly.

From the EUS image alone, we suspected that the mass was a malignant gastrointestinal mesenchymal tumor due to the lesion arising from the muscularis propria layer of the duodenum with a hypoechoic heterogeneous pattern and poorly defined border [10]. Stromal subepithelial tumors are a group of mesenchymal neoplasms that includes gastrointestinal stromal tumor (GIST) and non-GIST. GIST of more than $5 \mathrm{~cm}$ indicates malignancy, but the nature of the tumor may depend on its location as some reported that small-bowel GIST behave more aggressively [11].

Gastroptosis has rarely been reported in recent times, given that its clinical significance of remains unclear. Gastroptosis has never been reported to be caused by GOO and duodenal tumor, and similarly, GOO has never been reported to worsen gastroptosis.

\section{Acknowledgements}

The authors would like to thank American Journal Experts for English editing service.

\section{Statement of Ethics}

This retrospective review of patient data did not require ethical approval in accordance with local/national guidelines. Written informed consent was obtained from participant next-of-kin for publication of the details of this medical case and any accompanying images.

\section{Karger'}


Bestari et al.: Gastroptosis due to Gastric Outlet Obstruction Secondary to Duodenal Tumor

\section{Conflict of Interest Statement}

The authors have no conflicts of interest to declare.

\section{Funding Sources}

This case report did not receive funding support.

\section{Author Contributions}

M.B.B., I.R.J., and M.C. wrote the manuscript. M.B.B., M.C., D.G., and R.A. cared for the patient. Y.W. performed the endoscopy, and S.A.A. critically reviewed and revised the manuscript. All the authors read and approved the final manuscript.

\section{Data Availability Statement}

All data generated or analyzed during this study are included in this article. Further inquiries can be directed to the corresponding author.

\section{References}

1 Aaron CD. Gastroptosis. JAMA. 1897;XXIX(5):224.

2 Ban T, Kawakami H, Kubota Y, Nanashima A, Yano K, Sato Y. The development of gastric outlet obstruction due to a lumen-occupying protruding duodenal ulcer mimicking a submucosal tumor. Intern Med. 2019 May 1; 58(9):1267-71.

3 Beilin DS. When has visceroptosis clinical significance? Radiology. 1930;15(2):223-6.

4 Sukimo S, Kazutaka $\mathrm{H}$. The forms of the stomach in healthy individuals : with special reference to analysis of relationships between the degree of gastroptosis and sex, age, comparative weight ratio or equivocal complaints. Kitasato Med. 1986;16(4):211-26.

5 Sullivan SN. Gastroptosis: a cause of postprandial abdominal bloating. Can J Gastroenterol Hepatol. 1991;5:3.

6 Sarangapani A, Rasane S, Kohli V, Chandy G. Glenard's disease. Arch Med Health Sci. 2016;4(1):153-4.

7 Christianakis E, Bouchra K, Koliatou A, Paschalidis N, Filippou D. Gastroparesis associated with gastroptosis presenting as a lower abdominal bulking mass in a child: a case report. Cases J. 2009 Nov 4;2:184.

8 Hassan SM, Mubarik A, Muddassir S, Haq F. Adult idiopathic hypertrophic pyloric stenosis: a common presentation with an uncommon diagnosis. J Community Hosp Intern Med Perspect. 2018;8(2):64-7.

9 Maglinte DD, Kelvin FM, Rowe MG, Bender GN, Rouch DM. Small-bowel obstruction: optimizing radiologic investigation and nonsurgical management. Radiology. 2001 Jan;218(1):39-46.

10 Pavlovic Markovic A, Rosch T, Alempijevic T, Krstic M, Tomic D, Dugalic P, et al. Endoscopic ultrasound for differential diagnosis of duodenal lesions. Ultraschall in der Medizin. 2012 Dec;33(7):E210-17.

11 Miettinen M, El-Rifai W, Sobin LHL, Lasota J. Evaluation of malignancy and prognosis of gastrointestinal stromal tumors: a review. Human Pathol. 2002 May;33(5):478-83. 